\title{
Performance Analysis of a Persistent Relay Carrier Sensing Multiple Access Protocol
}

\author{
J. Alonso-Zárate, Member, IEEE, L. Alonso, Member, IEEE, and Ch. Verikoukis, Senior Member, IEEE
}

\begin{abstract}
The Persistent Relay Carrier Sensing Multiple Access (PRCSMA) has been recently presented in the literature as a novel Medium Access Control (MAC) protocol based on the IEEE 802.11 and modified to cope with the contention among the relays in a Cooperative ARQ (C-ARQ) scheme. We present in this paper a theoretical model to calculate the average cooperation delay of PRCSMA. Computer simulations have been carried out to assess the accuracy of the model.
\end{abstract} ysis.

Index Terms-PRCSMA, cooperative ARQ, performance anal-

\section{INTRODUCTION}

$\mathbf{C}$ OOPERATIVE Automatic Retransmission reQuest (CARQ) schemes have been proposed as an efficient way to exploit the broadcast nature of the wireless channel in order to improve the performance of wireless networks [1]. In a C-ARQ scheme, communication takes place in at least four phases when a packet is received with errors at the destination. In the first phase, the source transmits a data packet to the destination. In the second phase, the destination broadcasts a cooperation request message, thus initiating a cooperation phase. Some of the stations which received the original transmission from the source and also receive this cooperation request message become spontaneous relays and can provide the communication with cooperative diversity. In the third phase, the relays retransmit a copy of the original transmission to the destination. Finally, in the fourth phase, the destination acknowledges (either positively or negatively) the reception of the packet to the source, also announcing the end of the cooperation phase.

The benefits of C-ARQ from the physical (PHY) layer point of view have been discussed in detail in [1]-[5]. These works demonstrate that C-ARQ schemes can improve the performance of wireless communications in terms of throughput, delay, energy consumption, or even coverage extension. However, the contention problem among the relays is not considered in most of the cases and a perfect retransmission scheduling is usually assumed. The need to lift these assumptions and to design a more realistic C-ARQ is the main motivation for the work presented in this paper.

Manuscript received May 15, 2009; revised August 10, 2009; accepted September 23, 2009. The associate editor coordinating the review of this letter and approving it for publication was J. Zhang.

This work has been supported by LOOP (FIT- 330215-2007-8), NEWCOM++ (ICT-216715), PERSEO (TEC2006-10459/TCM), COOLNESS (218163-FP7-PEOPLE-2007-3-1-IAPP), and CENTENO (TEC2008-06817C02-02).

J. Alonso-Zárate and C. Verikoukis are with the Centre Tecnològic de Telecomunicacions de Catalunya (CTTC), Castelldefels, Barcelona, Spain (email: \{jalonso, cveri\}@cttc.es).

L. Alonso is with the Dept. of Signal Theory and Communications, Universitat Politècnica de Catalunya (UPC-EPSC), Castelldefels, Barcelona, Spain (e-mail: luisg@tsc.upc.edu).

Digital Object Identifier 10.1109/TWC.2009.12.090707
We focus on the Medium Access Control (MAC) layer of C-ARQ schemes. Within this context, the cooperation delay can be defined as the time elapsed from the moment a station requests cooperation until it can acknowledge the data packet that had been originally received with errors by combining a number of retransmitted copies received from the relays. An accurate estimation of the average of this delay can be very useful in order to decide upon initiating a cooperation phase or not each time a packet is received with errors. Under some conditions, it may be more convenient to discard a packet for the benefit of the backlogged data rather than initiating a cooperation phase.

To the best of our knowledge, the Persistent Relay Carrier Sensing Multiple Access (PRCSMA) [6] is one of the few MAC protocols that have been designed to coordinate the retransmissions of the relays in a C-ARQ scheme. PRCSMA is based on the IEEE 802.11 MAC protocol [7] and the average cooperation delay of a system executing this MAC was calculated in [6] using the existing models of Bianchi [8], Wu et al. [9], and Chatzimisios et al. [10] for the IEEE 802.11 MAC protocol. Since these models analyze the saturation throughput of an IEEE 802.11 network, the analysis of PRCSMA presented in [6] assumes that:

1) The same relays are available to cooperate each time a packet is received with errors. However, this may not be always feasible in a real network.

2) The relays have a dedicated backoff counter for the cooperation phases and another independent backoff counter for their own data transmissions.

3) The relays maintain the latest value of the backoff counter and use it for the next cooperation phase. However, in the original definition of PRCSMA it is specified that the relays reset the value of the backoff counter for each cooperation phase.

Our main contribution in this paper is to present a new theoretical model of PRCSMA that overcomes these limitations by considering that:

1) Not the same the relays might be available for each cooperation phase.

2) The relays do not need a dedicated backoff counter for the cooperation phases.

3) The relays reset the value of the backoff counter when a new cooperation phase is initiated.

The remainder of the paper is organized as follows. In Section II, we present an overview of the operation of PRCSMA. In Section III, we analyze the performance of PRCSMA. Section IV is devoted to validating the model through extensive computer simulation. Finally, Section V concludes the paper. 


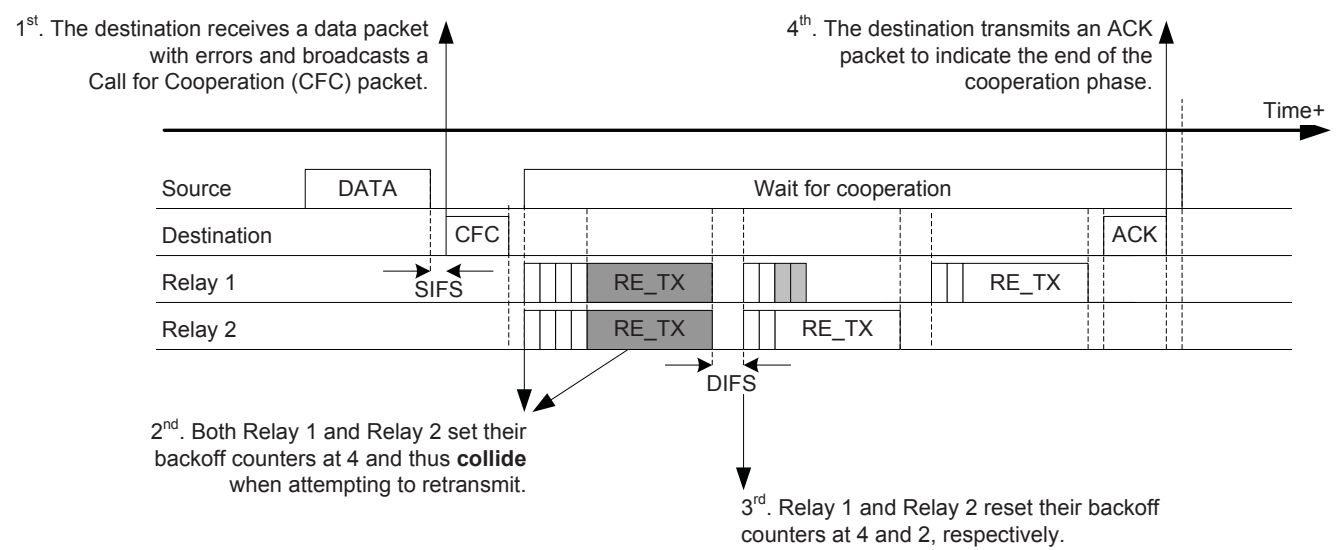

Fig. 1. Example: PRCSMA operation.

\section{PRCSMA OVERVIEW}

Consider a wireless network formed by an arbitrary number of stations equipped with half-duplex radio frequency transceivers. All the stations listen to every ongoing transmission in order to be able to cooperate if required. We assume that error detection bits (e.g., Cyclic Redundancy Codes (CRC)) are attached to all the transmitted data packets. Each time a destination receives a data packet with unrecoverable errors, it broadcasts a Call for Cooperation (CFC) packet, thus initiating a cooperation phase. Some of the stations which overheard the original data transmission from the source and receive the CFC from the destination become active relays or helpers. The specific relay selection criteria is out of the scope of the basic definition of PRCSMA. Orthogonally in time, the active relays attempt to retransmit an amplified, compressed, or recoded copy of the original packet to assist the failed transmission. The contention of the relays is based on the Distributed Coordination Function (DCF) defined in the IEEE 802.11 Standard [7], which is based on CSMA. When DCF is executed, all the stations listen to the channel before attempting to transmit for the first time. If the channel is idle, they transmit; otherwise a random backoff is initiated. To do so, they set the backoff counter to a random value in the interval $[0, C W]$, where $\mathrm{CW}$ represents the size of the contention window. As long as the channel is sensed idle, this counter is decremented by one unit after every time slot. Whenever the counter expires, i.e., hits zero, the station attempts a new transmission. In the case of collision or transmission failure, the value of $\mathrm{CW}$ is doubled up (up to a predefined maximum value) in order to reduce the probability of collision in subsequent transmission attempts. Whenever a transmission is successful, the value of $\mathrm{CW}$ is reset to the minimum size. Positive acknowledgments (ACK) are used to provide the transmitter with feedback. An optional Collision Avoidance (CA) mechanism is defined in the 802.11 MAC protocol by which a Request-to-Send / Clear-to-Send (RTS/CTS) handshake is established between source and destination prior to the the data transmission. The operation of PRCSMA is based on this algorithm but considering the four following modifications:

1) In order to avoid a certain first collision, active relays backoff before attempting to retransmit for the first time upon the reception of a CFC packet.
2) There is no ACK transmission associated to each retransmitted packet as the relays do not need feedback for each retransmission.

3) Relays never increase the size of their contention window as they do not have any mechanism to detect collisions unless they use the CA access mode with RTS/CTS handshake. However, in order to reduce the overhead, it is more convenient to use the basic access mode.

4) A cooperation phase finishes whenever the destination transmits an ACK packet to notify the successful decoding of the original data packet (by properly combining the different copies received from the relay set [11] or because an error-free copy has been received from a relay), or when it transmits a NACK packet if a certain maximum cooperation time-out has elapsed and the original packet has to be discarded.

In order to illustrate the operation of PRCSMA, an example of operation is presented in Fig. 1. In this example, a source and a destination are assisted by two relays. Initially, the source transmits a data packet to the destination. After a Short Inter Frame Space (SIFS), the destination broadcasts a CFC packet and a cooperation phase is initiated. Two of the stations which receive the CFC packet become active relays. We refer to these relays as Relay 1 and Relay 2 . Each of the two active relays selects, at random, a value for their respective backoff counters. In this example, they select the same value (4). Therefore, after 4 time slots, the two relays attempt to retransmit and a collision occurs. After a Distributed IFS (DIFS), the two relays reselect at random a new value for their backoff counters to attempt a new retransmission. In this case, Relay 1 selects the value 4 and Relay 2 selects the value 2 . Therefore, after two time slots, Relay 2 successfully retransmits a packet. After the completion of this retransmission, Relay 1 waits for the remaining backoff time (two slots) and for an additional DIFS time to ensure that no ACK has been sent by the destination, and then begins the packet retransmission. Finally, upon the reception of the second retransmission, the destination station is able to decode the original packet by properly combining the information of the original transmission from the source plus the two retransmissions from the relays. Accordingly, it transmits an ACK packet to acknowledge the reception of the original data packet and to advertise the end of the cooperation phase. 


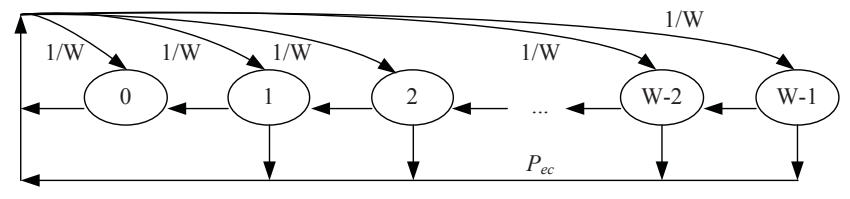

Fig. 2. PRCSMA embedded Markov chain.

\section{PERformance AnAlysis}

We consider the network formed by a source, a destination, and $n$ relays. The average packet transmission delay given that cooperation is executed is defined as

$$
\begin{aligned}
& \mathrm{E}\left[T_{D}\right]=T_{S}+ \\
& +\left[3 \cdot T_{S I F S}+T_{C F C}+K \cdot T_{R}+\mathrm{E}\left[T_{\text {cont }}\right]+T_{A C K}\right]
\end{aligned}
$$

where $T_{S}$ is the data transmission time from the source. The sum term in squared brackets corresponds to the average cooperation delay, defined as the time elapsed from the moment a packet is received with errors until it is acknowledged to the transmitter after receiving $K$ retransmissions. $T_{S I F S}$ is the duration of a SIFS, $T_{C F C}$ and $T_{A C K}$ are the transmission times of $\mathrm{CFC}$ and $\mathrm{ACK}$ packets, respectively, and $T_{R}$ is the duration of a successful retransmission from the relays as reported in [6]. $\mathrm{E}\left[T_{\text {cont }}\right]$ is the average contention time (time spent in backoff and collisions) and its value depends on the specific MAC protocol. We compute the value of this parameter within the context of PRCSMA in the next subsection.

\section{A. PRCSMA Model}

The cooperation backoff counter of an individual relay can be modeled with the embedded Markov chain depicted in Fig. 2. Each of the $W$ states in the chain represents the current value of the backoff counter of the relay. The state 0 represents a transmission attempt by the relay. Independently of whether a success or failure occurs in this transmission, the relay resets its backoff counter right after transmitting. The backoff counter is also reset if the current cooperation phase is finished before attempting to transmit. This event happens with probability $P_{e c}$. Note that this probability was not considered in the analysis presented in [6], as in that work it was assumed that the relays use the last value of the backoff counter for a new cooperation phase. We consider a slottedtime reference where the time slot is the unit of time between consecutive backoff counter decrements. We denote $P_{k}$ the steady state probability of being in state $k$ in a given slot and, by observation of the chain, it is possible to write

$$
\begin{aligned}
& P_{W-1}=\frac{1}{W}\left(P_{0}+\sum_{k=1}^{W-1} P_{k} P_{e c}\right) \\
& P_{W-2}=\frac{1}{W}\left(P_{0}+\sum_{k=1}^{W-1} P_{k} P_{e c}\right)+P_{W-1}\left(1-P_{e c}\right) \\
& P_{W-3}=\frac{1}{W}\left(P_{0}+\sum_{k=1}^{W-1} P_{k} P_{e c}\right)+P_{W-2}\left(1-P_{e c}\right) \\
& P_{W-4}=\ldots
\end{aligned}
$$

Note that the probability that a station attempts to transmit in a given slot is $P_{0}$ (when the backoff counter expires).
Equation (2) can be generalized for any state $k$ as

$$
P_{k}=\sum_{j=0}^{(W-1)-k} \frac{1}{W}\left(P_{0}+\sum_{i=1}^{W-1} P_{i} P_{e c}\right)\left(1-P_{e c}\right)^{j}
$$

The sum of all the probabilities of the states must be equal to one and thus

$$
\sum_{k=0}^{W-1}\left[\frac{1}{W}\left(P_{0}+\left(1-P_{0}\right) P_{e c}\right) \sum_{j=0}^{(W-1)-k}\left(1-P_{e c}\right)^{j}\right]=1 .
$$

After some algebra, the value of $P_{0}$ can be expressed as

$$
P_{0}=\frac{1}{\left(1-P_{e c}\right)}\left[\frac{W-P_{e c} \sum_{k=0}^{W-1} \sum_{j=0}^{(W-1)-k}\left(1-P_{e c}\right)^{j}}{\sum_{k=0}^{W-1} \sum_{j=0}^{(W-1)-k}\left(1-P_{e c}\right)^{j}}\right] .
$$

If there is only one active relay, it holds that $P_{e c}=0$, and thus $P_{0}$ can be rewritten as

$$
P_{0}=\frac{W}{\sum_{k=0}^{W-1} \sum_{j=0}^{W-1)-k} 1^{j}}=\frac{2 W}{W^{2}+W-2} .
$$

Otherwise, if there is more than one active relay, the value of $P_{0}$ expressed in (5) can be simplified as follows. We use $j=W-k$ and the fact that for any $0<a<1$ it holds that $\sum_{n=0}^{N} a^{n}=\left(1-a^{N+1}\right) /(1-a)$ to rewrite the sum terms in $(5)$ as

$$
\begin{aligned}
\sum_{k=0}^{W-1} \sum_{j=0}^{(W-1)-k}\left(1-P_{e c}\right)^{j}= & \frac{\left(W P_{e c}-1+\left(1-P_{e c}\right)^{W+1}+P_{e c}\right)}{P_{e c}^{2}} .
\end{aligned}
$$

Then, the value of $P_{0}$ can be computed as

$$
P_{0}=\frac{1}{\left(1-P_{e c}\right)}\left[\frac{P_{e c}\left(1-P_{e c}-\left(1-P_{e c}\right)^{W+1}\right)}{(W+1) P_{e c}-1+\left(1-P_{e c}\right)^{W+1}}\right] \text {. }
$$

We intentionally leave the calculation of $P_{e c}$ for the next subsection, where we compute the average contention delay of PRCSMA.

\section{B. Average Contention Delay Analysis}

Due to the fully distributed operation of PRCSMA, the contention time of a packet is independent of the contention time of any other packet (from the network perspective). Therefore, the average contention time of PRCSMA can be computed as

$$
\mathrm{E}\left[T_{\text {cont }}\right]=K \cdot \mathrm{E}[X] \cdot \mathrm{E}\left[T_{n s s}\right],
$$

where $\mathrm{E}[X]$ denotes the average number of non-successful slots (i.e., slots that contain a collision, a transmission error, or simply idle slots) required to achieve a successful transmission and $\mathrm{E}\left[T_{n s s}\right]$ denotes the average duration of a non-successful slot. We define $P_{s}$ as the probability of having a successful 
transmission in a given slot, and thus $\mathrm{E}[X]$ corresponds to the mean value of a geometric distribution minus one (we subtract the successful slot). Therefore,

$$
\mathrm{E}[X]=\left[\sum_{X=0}^{\infty} X\left(1-P_{S}\right)^{(X-1)} P_{S}\right]-1=\frac{1}{P_{S}}-1 .
$$

On the other hand, and applying Bayes' theorem, $\mathrm{E}\left[T_{n s s}\right]$ can be computed as

$$
\mathrm{E}\left[T_{n s s}\right]=\left(\frac{P_{I}}{1-P_{S}}\right) \sigma+\left(\frac{P_{E}}{1-P_{S}}\right) T_{R}+\left(\frac{P_{C}}{1-P_{S}}\right) T_{C} .
$$

$\sigma$ is the duration of an idle slot as defined at the PHY layer [7] and $T_{C}$ is the duration of a collision as given in [6]. In addition, since a cooperation phase finishes after $K$ successful retransmissions and a successful transmission occurs, in average, every $\mathrm{E}[X]$ slots, then

$$
P_{e c}=(K \cdot \mathrm{E}[X])^{-1}=\left(P_{S} / K\right) .
$$

Finally, $P_{I}, P_{E}$, and $P_{C}$ are the probabilities of having an idle slot, a transmission error or a collision, respectively. In order to compute them, we first define $p$ as the probability of transmission failure (collision or transmission error) perceived by any of the $n$ relays when attempting to transmit in a given slot. As in [8]-[10], we assume that $p$ has a constant value that, in the presence of transmission errors, can be computed as

$$
p=1-\left[\left(1-P_{0}\right)^{n-1}\left(1-p_{e}\right)\right],
$$

with $p_{e}$ the probability that a transmission error occurs. On the other hand, the probability that at least one relay attempts to transmit in a given slot is denoted by $P_{t r}$ and can be expressed as

$$
P_{t r}=1-\left(1-P_{0}\right)^{n} .
$$

Further, the probability of having a single transmission in a slot given that at least one station transmits is denoted by $p_{s}$ and can be expressed as

$$
p_{s}=\left(n P_{0}\left(1-P_{0}\right)^{n-1}\right) / P_{t r} .
$$

Finally, the values of $P_{I}, P_{S}, P_{E}$, and $P_{C}$ can be written as

$$
\begin{aligned}
& P_{I}=1-P_{t r}, \\
& P_{S}=P_{t r} p_{s}\left(1-p_{e}\right), \\
& P_{E}=P_{t r} p_{s} p_{e} \\
& P_{C}=P_{t r}\left(1-p_{s}\right) .
\end{aligned}
$$

At this point in the analysis, we have all the terms required to compute (9) and, therefore, to obtain the value of the average packet transmission delay when cooperation is requested as expressed in (1). We evaluate the accuracy of this model in the next section.

\section{Model Validation and Performance EVALUATION}

The formulas in Section III have been used to obtain numerical results. These results have been compared to the ones obtained with link-level computer simulations in MATLAB where the actual protocol operation has been implemented. This comparison validates the accuracy of the analytical
TABLE I

SYSTEM PARAMETERS

\begin{tabular}{|c|c|c|c|}
\hline Parameter & Value & Parameter & Value \\
\hline \hline MAC header & 34 bytes & PHY preamble & $96 \mu s$ \\
\hline Data Tx. Rate S-D & $6 / 24 \mathrm{Mbps}$ & Ctrl. Tx. Rate S-D & $6 \mathrm{Mbps}$ \\
\hline Data Tx. Rate R-D & $54 \mathrm{Mbps}$ & Ctrl. Tx. Rate R-D & $6 \mathrm{Mbps}$ \\
\hline ACK length & 14 bytes & Data Packets & 1500 bytes \\
\hline RTS length & 20 bytes & CTS length & 14 bytes \\
\hline CFC length & 14 bytes & $C W$ & 32 \\
\hline DIFS & $50 \mu s$ & SIFS and $\sigma$ & $10 \mu \mathrm{s}$ \\
\hline
\end{tabular}

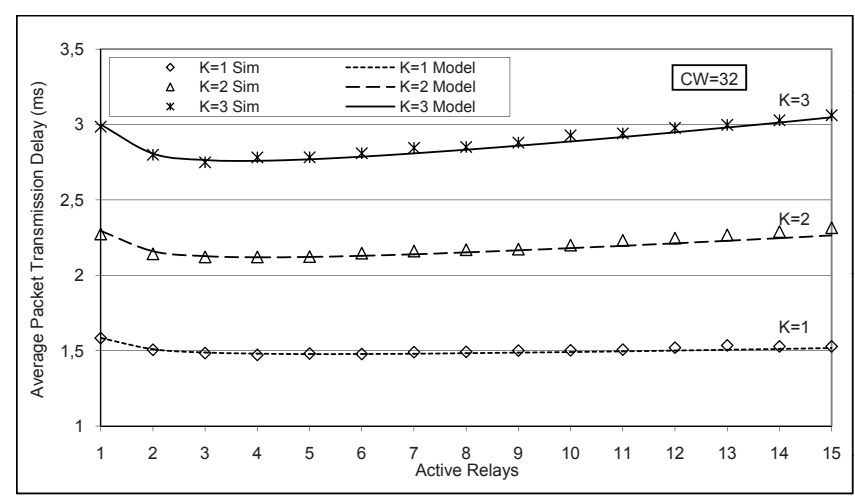

Fig. 3. Model vs. simulation PRCSMA (CW=32).

calculations presented in the previous section. The values of the parameters used for both formulas and the simulations are based on the PHY layer of the IEEE 802.11g Standard for WLANs [7] and they are summarized in Table I. Sequential batches of cooperation phases have been simulated and the results have been averaged to ensure statistical independence. Different experiments have been carried out considering different number of relays for each cooperation phase (from 1 to 15). We consider in all cases that the relays use the basic access mode of PRCSMA [6], i.e., without RTS/CTS.

First, the accuracy of the proposed model is shown in Fig. 3 where the results of the simulations and the theoretical model are plotted together. The average packet transmission delay is represented as a function of the number of active relays, for different values of $K$ (number of required retransmissions), and considering $C W=32$. The lines (model) and the markers (simulation) show an almost perfect match. As far as performance is concerned, it can be observed that the average packet transmission delay is more sensitive to the number of active relays as the value of $K$ grows. As it could be expected, the more retransmissions required, the longer the contention period among the relays, and thus the larger the MAC overhead. However, irrespectively of the value of $K$, there always exist an optimum number of active relays which minimizes the average delay. Indeed, this optimum number of relays also depends on the value of $\mathrm{CW}$. For a given $\mathrm{CW}$, the probability of collision increases with the number of relays. Therefore, if the value of $\mathrm{CW}$ is small compared to the number of relays, the probability of collision is high and the average delay increases. On the other hand, if the value of $\mathrm{CW}$ is big, there will be an unnecessary waste of time devoted to backoff deferral periods. In order to better show this phenomenon, the average packet transmission delay for different sizes of CW is depicted in Fig. 4 when $K=3$ (although the results are 


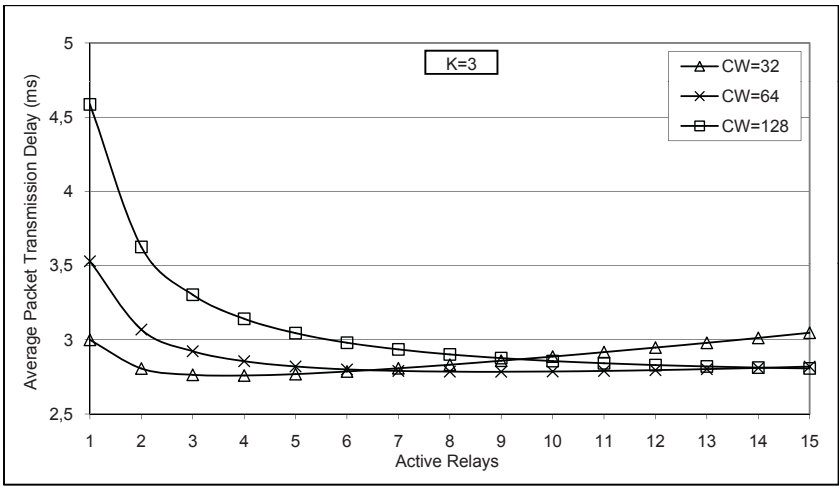

Fig. 4. PRCSMA with different sizes of the CW.

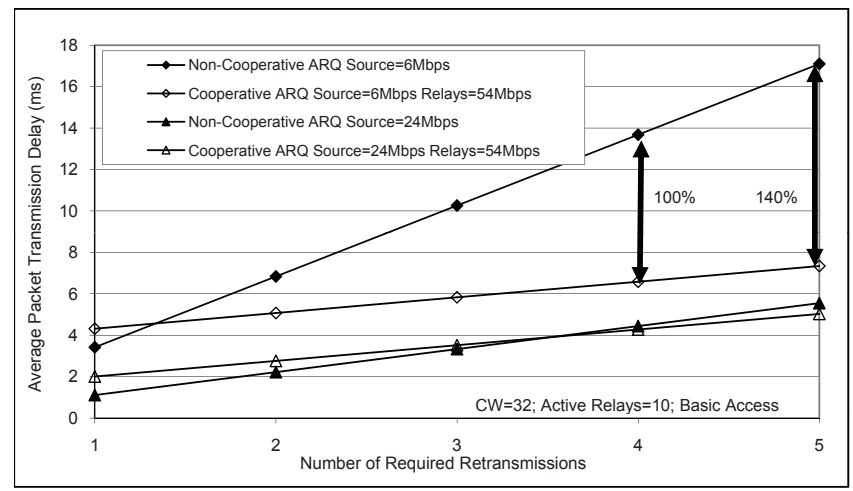

Fig. 5. C-ARQ with PRCSMA vs. non-cooperative ARQ.

similar for any value of $K$ ). A small value of CW yields better performance when the number of relays is low while larger values of $\mathrm{CW}$ perform better as the number of relays is high. However, as the number of relays becomes high, the selection of the size of $\mathrm{CW}$ becomes less critical. Indeed, there are remarkable differences in the average delay for different values of $\mathrm{CW}$ when the number of relays is low, but these differences are reduced as the number of relays grows. Therefore, the optimum design of $\mathrm{CW}$ becomes a key factor when the relay selection criterion is restrictive and the number of actual active relays is small. Otherwise, a mistuning of the value of $\mathrm{CW}$ may just yield a slightly higher average packet transmission delay when cooperation is required.

For completeness, the average packet transmission delay of a C-ARQ scheme using PRCSMA is compared to that of a non-cooperative ARQ scheme in Fig. 5. For the noncooperative ARQ case we have considered that all the retransmissions are performed only from the source. We have considered two different data transmission rates for the source while the transmission rate at the control plane has been fixed to $6 \mathrm{Mbps}$ in all cases. The results in Fig. 5 show that there is a tradeoff between the faster retransmissions of the relays and the overhead required to coordinate these retransmissions. When the transmission rate of the relays is about twice the transmission rate of the source (54 and $24 \mathrm{Mbps}$ in this case, respectively), the C-ARQ scheme outperforms the noncooperative ARQ only when $K>4$. However, in the case that the transmission rate of the relays is much higher than that of the source (54 and $6 \mathrm{Mbps}$, respectively) the CARQ outperforms the non-cooperative ARQ remarkably just when $K>2$. Note that, for example, the average packet transmission delay is divided by 2 when $K=4$. Finally, if the transmission rate of the relays is comparable to (or even lower than) that of the source, there are no gains in terms of delay as cooperation introduces extra coordination overhead. In these cases, cooperation can be useful in terms of diversity or energy efficiency, but not in terms of delay.

\section{CONCLUSIONS}

In this paper, we have presented a theoretical model for the calculation of the average cooperation delay of PRCSMA (a MAC protocol for C-ARQ). The validity of the proposed model has been demonstrated with the help of link-level computer simulations. Results show that, for a given MAC configuration, there is an optimum number of relays that should cooperate in order to minimize the cooperation delay taking into account the actual contention among relays. In addition, the comparison of the performance of a C-ARQ scheme with a non-cooperative ARQ shows that if the relays cannot transmit at higher transmission rates than the source, the MAC overhead required to coordinate the relays reduces the efficiency of cooperation. Therefore, the design of efficient MAC protocols that can reduce the overhead required to coordinate the retransmissions from the relays constitutes a challenging open topic for future research.

\section{ACKNOWLEDGMENT}

The authors would like to thank the reviewers for their useful comments as well as E. Kartsakli and A. Antonopoulos for their valuable support in preparing the final version of the paper.

\section{REFERENCES}

[1] M. Dianati, X. Ling, K. Naik, and X. Shen, "A node-cooperative ARQ scheme for wireless ad hoc networks," IEEE Trans. Veh. Technol., vol. 46, pp. 1032-1044, May 2006.

[2] E. Zimmermann, P. Herhold, and F. Fettweis, "The impact of cooperation on diversity-exploiting protocols," in Proc. VTC, 2004.

[3] _ _ "On the performance of cooperative relaying protocols in wireless networks," European Trans. Commun., vol. 16, pp. 5-16, Jan. 2005.

[4] I. Cerruti, A. Fumagalli, and P. Gupta, "Delay model of single-relay cooperative ARQ protocols in slotted radio network with Poisson frame arrivals," IEEE/ACM Trans. Networking, vol. 16, pp. 371-382, Apr. 2008.

[5] J. Morillo-Pozo, J. García-Vidal, and A. I. Pérez-Neira, "Collaborative ARQ in wireless energy-constrained networks," in Proc. Joint Workshop on Foundations of Mobile Computing, DIAL-POM, 2005.

[6] J. Alonso-Zárate, E. Kartsakli, C. Verikoukis, and L. Alonso, "Persistent RCSMA: a MAC protocol for a distributed cooperative ARQ scheme in wireless networks," EURASIP J. Advanced Signal Processing, special issue on wireless cooperative networks, p. 13, Dec. 2008.

[7] Wireless LAN Medium Access Control (MAC) and Physical Layer (PHY) Specifications, IEEE Standard 802.11-2007.

[8] G. Bianchi, "Performance analysis of the IEEE 802.11 distributed coordination function," IEEE J. Sel. Areas Commun., vol. 18, pp. 535547, Mar. 2000.

[9] H. Wu, Y. Peng, K. Long, S. Cheng, and J. Ma, "Performance of reliable transport protocol over IEEE 802.11 wireless LAN: analysis and enhancement," in Proc. INFOCOM 2002, vol. 2, pp. 599-607.

[10] P. Chatzimisios, A. Boucouvalas, and V. Vitsas, "Performance analysis of IEEE 802.11 DCF in presence of transmission errors," in Proc. IEEE International Conference on Communications (ICC), vol. 7, June 2004, pp. 3854-3858.

[11] J. D. Morillo and J. García-Vidal, "A low coordination overhead CARQ protocol with frame combining," in Proc. Symposium on Personal, Indoor and Radio Communications (PIMRC), 2007. 